\title{
Existence results for a variable exponent elliptic problem via topological method
}

\section{Shifeng Yang and Guowei Dai*}

"Correspondence: daiguowei@nwnu.edu.cn

School of Finance, Lanzhou University of Finance and

Economics, Lanzhou, 730020, P.R. China

\begin{abstract}
In this paper, existence, localization and uniqueness results of solutions to elliptic Dirichlet boundary value problems are established. The approach is based on the nonlinear alternative of Leray-Schauder, the Brouwer fixed point theorem and the Galerkin method.
\end{abstract}

MSC: $35 \mathrm{~J} 60 ; 47 \mathrm{H} 10$

Keywords: $p(x)$-Laplacian; Leray-Schauder principle; fixed point; Galerkin method

\section{Introduction}

In this paper, we consider the boundary value problem

$$
\left\{\begin{array}{l}
-\operatorname{div}\left(|\nabla u|^{p(x)-2} \nabla u\right)=f(x, u) \quad \text { in } \Omega, \\
u=0 \quad \text { on } \partial \Omega,
\end{array}\right.
$$

where $\Omega \subset \mathbb{R}^{N}$ is a nonempty bounded open set with smooth boundary $\partial \Omega, p=p(x) \in$ $C_{+}(\bar{\Omega})$ with $1<p^{-}:=\min _{\bar{\Omega}} p(x) \leq p^{+}:=\max _{\bar{\Omega}} p(x)<+\infty$ and $f: \Omega \times \mathbb{R} \rightarrow \mathbb{R}$ is a continuous function.

The operator $-\operatorname{div}\left(|\nabla u|^{p(x)-2} \nabla u\right)$ is said to be the $p(x)$-Laplacian and becomes $p$ Laplacian when $p(x) \equiv p$ (a constant). The $p(x)$-Laplacian possesses more complicated nonlinearities than the $p$-Laplacian; for example, it is inhomogeneous. The study of various mathematical problems with a variable exponent growth condition has received considerable attention in recent years. These problems are interesting in applications and raise many difficult mathematical problems. One of the most studied models leading to a problem of this type is the model of motion of electro-rheological fluids, which are characterized by their ability to drastically change the mechanical properties under the influence of an exterior electro-magnetic field [1,2]. Problems with variable exponent growth conditions also appear in the mathematical modeling of stationary thermo-rheological viscous flows of non-Newtonian fluids and in the mathematical description of the processes filtration of an ideal baro-tropic gas through a porous medium [3, 4]. Another field of application of equations with variable exponent growth conditions is image processing [5]. The variable nonlinearity is used to outline the borders of the true image and to eliminate possible noise. We refer the reader to [6-11] for an overview of and references on this subject, and to [12-21] for the study of the $p(x)$-Laplacian equations and the corresponding variational problems.

\section{空 Springer}

(C) 2012 Yang and Dai; licensee Springer. This is an Open Access article distributed under the terms of the Creative Commons Attribution License (http://creativecommons.org/licenses/by/2.0), which permits unrestricted use, distribution, and reproduction in any medium, provided the original work is properly cited. 
In recent years, many authors have studied the existence of solutions for problem (1.1) from several points of view and with different approaches (see, for example, [18-20]). A useful method for the investigation of solutions to semilinear problems is based on the Leray-Schauder continuation principle, or equivalently, on Schaefers fixed point theorem. For example, in [22] this method was used for solutions in Hölder spaces, while in [23], solutions were found in Sobolev spaces.

The aim of this paper is to present new existence, localization and uniqueness results for solutions to problem (1.1) under suitable conditions on the nonlinearity $f$. Our approach is based on regularity results for the solutions of linear Dirichlet problems, the nonlinear alternative of Leray-Schauder (see [24]), the Brouwer fixed point theorem (see [25]) and the Galerkin method. We notice that our partial results of the present paper are motivated by the papers [26] and [27] where the authors have obtained some results for semilinear and quasilinear elliptic boundary value problems, respectively. By the Galerkin method, we also establish the results of existence and uniqueness of a solution for problem (1.1). We also would like to point out that the proof of Theorem 3 of [27] is wrong since $\left(-\Delta_{p}\right)^{-1}$ is not a linear operator. In this paper, we give a key lemma that can be used to overcome this difficulty.

The rest of this paper is organized as follows. In Section 2, we present some necessary preliminary knowledge on variable exponent Sobolev spaces. In Section 3, we shall use a nonlinear alternative of Leray-Schauder to prove the existence of solutions for problem (1.1). In Section 4, by the Galerkin method, we shall establish the results of existence and uniqueness of a solution for problem (1.1).

\section{Preliminaries}

In order to discuss problem (1.1), we need some theories on $W_{0}^{1, p(x)}(\Omega)$ which we call a variable exponent Sobolev space. Firstly, we state some basic properties of spaces $W_{0}^{1, p(x)}(\Omega)$ which will be used later (for details, see [7]). Denote by $\mathbf{S}(\Omega)$ the set of all measurable real functions defined on $\Omega$.

Write

$$
C_{+}(\bar{\Omega})=\{h: h \in C(\bar{\Omega}), h(x)>1 \text { for any } x \in \bar{\Omega}\}
$$

and

$$
L^{p(x)}(\Omega)=\left\{u \in \mathbf{S}(\Omega): \int_{\Omega}|u(x)|^{p(x)} d x<+\infty\right\}
$$

with the norm

$$
|u|_{L^{p(x)}(\Omega)}=|u|_{p(x)}=\inf \left\{\lambda>0: \int_{\Omega}\left|\frac{u(x)}{\lambda}\right|^{p(x)} d x \leq 1\right\},
$$

and

$$
W^{1, p(x)}(\Omega)=\left\{u \in L^{p(x)}(\Omega):|\nabla u| \in L^{p(x)}(\Omega)\right\}
$$

with the norm

$$
\|u\|_{W^{1, p(x)}(\Omega)}=|u|_{L^{p(x)}(\Omega)}+|\nabla u|_{L^{p(x)}(\Omega)} \cdot
$$


Denote by $W_{0}^{1, p(x)}(\Omega)$ the closure of $C_{0}^{\infty}(\Omega)$ in $W^{1, p(x)}(\Omega)$. The spaces $L^{p(x)}(\Omega), W^{1, p(x)}(\Omega)$ and $W_{0}^{1, p(x)}(\Omega)$ are separable and reflexive Banach spaces.

Proposition 2.1 (See [7]) Set $\rho(u)=\int_{\Omega}|u(x)|^{p(x)} d x$. For any $u \in L^{p(x)}(\Omega)$, then

(1) for $u \neq 0,|u|_{p(x)}=\lambda \Leftrightarrow \rho\left(\frac{u}{\lambda}\right)=1$;

(2) $|u|_{p(x)}<1(=1 ;>1) \Leftrightarrow \rho(u)<1(=1 ;>1)$;

(3) if $|u|_{p(x)}>1$, then $|u|_{p(x)}^{p^{-}} \leq \rho(u) \leq|u|_{p(x)}^{p^{+}}$;

(4) if $|u|_{p(x)}<1$, then $|u|_{p(x)}^{p^{+}} \leq \rho(u) \leq|u|_{p(x)}^{p^{-}}$;

(5) $\lim _{k \rightarrow+\infty}\left|u_{k}\right|_{p(x)}=0 \Longleftrightarrow \lim _{k \rightarrow+\infty} \rho\left(u_{k}\right)=0$;

(6) $\lim _{k \rightarrow+\infty}\left|u_{k}\right|_{p(x)}=+\infty \Longleftrightarrow \lim _{k \rightarrow+\infty} \rho\left(u_{k}\right)=+\infty$.

In $W_{0}^{1, p(x)}(\Omega)$ the Poincaré inequality holds, that is, there exists a positive constant $C_{0}$ such that

$$
|u|_{L^{p(x)}(\Omega)} \leq C_{0}|\nabla u|_{L^{p(x)}(\Omega)}, \quad \forall u \in W_{0}^{1, p(x)}(\Omega)
$$

So, $|\nabla u|_{L^{p(x)}(\Omega)}$ is a norm equivalent to the norm $\|u\|_{W^{1, p(x)}(\Omega)}$ in the space $W_{0}^{1, p(x)}(\Omega)$. We shall use the equivalent norm in the following discussion and write $\|u\|=|\nabla u|_{L^{p(x)}(\Omega)}$ for simplicity.

Proposition 2.2 (1) [17, Theorem 4.1] Iff satisfies the sub-critical growth condition

$$
|f(x, t)| \leq c\left(1+|t|^{q(x)-1}\right), \quad \forall x \in \Omega, \forall t \in \mathbb{R}
$$

where $q \in C_{+}(\bar{\Omega})$ and $q(x)<p^{*}(x), \forall x \in \bar{\Omega}$, where $p^{*}(x)$ is the Sobolev critical exponent $\left(p^{*}(x)=N p(x) /(N-p(x))\right.$ if $p(x)<N$ and $p^{*}=+\infty$ if $\left.p(x) \geq N\right)$, then $u \in L^{\infty}(\Omega)$ for every weak solution $u$ of (1.1).

(2) [17, Theorem 4.4] Let $u \in W_{0}^{1, p(x)}(\Omega) \cap L^{\infty}(\Omega)$ be a solution of (1.1). If the function $p$ is log-Hölder continuous on $\bar{\Omega}$, i.e., there is a positive constant $H$ such that

$$
|p(x)-p(y)| \leq \frac{H}{-\log |x-y|} \quad \text { for } x, y \in \bar{\Omega} \text { with }|x-y| \leq \frac{1}{2}
$$

then $u \in C^{0, \alpha}(\bar{\Omega})$ for some $\alpha \in(0,1)$.

(3) [21, Theorem 1.2] If in (2), the condition (2.2) is replaced by that $p$ is Hölder continuous on $\bar{\Omega}$, then $u \in C^{1, \alpha}(\bar{\Omega})$ for some $\alpha \in(0,1)$.

From [20], we know that, for a given $h(x) \in L^{\frac{q(x)}{q(x)-1}}(\Omega)$, where $q(x)$ satisfies (2.1), the problem

$$
\left\{\begin{array}{l}
-\operatorname{div}\left(|\nabla u|^{p(x)-2} \nabla u\right)=h(x) \quad \text { in } \Omega \\
u=0 \text { on } \partial \Omega
\end{array}\right.
$$

has a unique solution $u \in W_{0}^{1, p(x)}(\Omega)$. We denote by $K(h):=u$ the unique solution. $K$ is called the solution operator for problem (2.3). It is well known that the solution operator $K$ is increasing (see Remark 2.1 of [18]). From the Proposition 2.2 and the embedding theorems, we can obtain the properties of the solution operator $K$ as follows. 
Proposition 2.3 (1) (See [20]) The mapping $K: L^{\frac{q(x)}{q(x)-1}}(\Omega) \rightarrow W_{0}^{1, p(x)}(\Omega)$ is continuous. Moreover, the mapping $K: L^{\frac{q(x)}{q(x)-1}}(\Omega) \rightarrow L^{q(x)}(\Omega)$ is completely continuous since the embedding $W_{0}^{1, p(x)}(\Omega) \rightarrow L^{q(x)}(\Omega)$ is compact.

(2) (See [17]) If $p$ is log-Holder continuous on $\bar{\Omega}$, then the mapping $K: L^{\infty}(\Omega) \rightarrow C^{0, \alpha}(\bar{\Omega})$ is bounded, and hence the mapping $K: L^{\infty}(\Omega) \rightarrow C(\bar{\Omega})$ is completely continuous.

(3) (See [21]) If $p$ is Hölder continuous on $\bar{\Omega}$, then the mapping $K: L^{\infty}(\Omega) \rightarrow C^{1, \alpha}(\bar{\Omega})$ is bounded, and hence the mapping $K: L^{\infty}(\Omega) \rightarrow C^{1}(\bar{\Omega})$ is completely continuous.

We note that the method of [26] cannot be directly used in this paper since $K$ is not a linear operator. So, we give a key lemma that will be used in Section 3 to overcome this difficulty.

Lemma 2.1 Let $M$ be a positive constant and $h(x) \in L^{\frac{q(x)}{q(x)-1}}(\Omega)$, then there exists one point $\xi \in \Omega$ such that

$$
K(M h)=M^{\frac{1}{p(\xi)-1}} K(h),
$$

where $K$ is the solution operator for problem (2.3).

Proof We assume that $u$ is a solution of problem (2.3), then we have $u=K(h)$. From (2.3), we can also show that

$$
\int_{\Omega} \frac{1}{p(x)}|\nabla u|^{p(x)} d x=\int_{\Omega} h(x) u(x) d x .
$$

By (2.4) and mean value theorem, for any positive constant $C$, we can show that there exists one point $\xi \in \Omega$ such that

$$
\begin{aligned}
\int_{\Omega} \frac{1}{p(x)}|\nabla(C u)|^{p(x)} d x & =C^{p(\xi)} \int_{\Omega} \frac{1}{p(x)}|\nabla u|^{p(x)} d x \\
& =C^{p(\xi)} \int_{\Omega} h(x) u(x) d x \\
& =\int_{\Omega} C^{p(\xi)-1} h(x) C u(x) d x,
\end{aligned}
$$

that is to say,

$$
K\left(C^{p(\xi)-1} h\right)=C K(h) .
$$

Let $M=C^{p(\xi)-1}$, then $C=M^{\frac{1}{p(\xi)-1}}$.

\section{Existence of a solution via the alternative of Leray-Schauder}

Here and in the sequel, $E$ will denote the space

$$
C_{0}(\bar{\Omega})=\{u \in C(\bar{\Omega}): u=0 \text { on } \partial \Omega\}
$$

endowed with the sup-norm

$$
\|u\|_{0}=\sup _{x \in \bar{\Omega}}|u(x)| .
$$


Now, we state an existence and localization principle for problem (1.1).

Theorem 3.1 (1) Assume that $f$ satisfies (2.1), $p(x) \in C_{+}(\bar{\Omega})$ and there is a constant $r>0$, independent of $\lambda>0$, with

$$
|u|_{L q(x)(\Omega)} \neq r
$$

for any solution $u \in W_{0}^{1, p(x)}(\Omega)$ to

$$
\left\{\begin{array}{l}
-\operatorname{div}\left(|\nabla u|^{p(x)-2} \nabla u\right)=\lambda f(x, u) \quad \text { in } \Omega \\
u=0 \text { on } \partial \Omega
\end{array}\right.
$$

and for each $\lambda \in(0,1)$. Then the boundary value problem (1.1) has at least one solution $u \in W_{0}^{1, p(x)}(\Omega)$ with $|u|_{L^{q(x)}(\Omega)} \leq r$.

(2) Assume that $p(x)$ is log-Hölder continuous and there is a constant $r>0$, independent of $\lambda>0$, with

$$
\|u\|_{0} \neq r
$$

for any solution $u \in C^{0, \alpha}(\bar{\Omega})$ to (3.2) and for each $\lambda \in(0,1)$. Then the boundary value problem (1.1) has at least one solution $u \in C^{0, \alpha}(\bar{\Omega})$ with $\|u\|_{0} \leq r$.

(3) Assume that $p(x)$ is Hölder continuous and there is a constant $r>0$, independent of $\lambda>0$, with (3.3) for any solution $u \in C^{1, \alpha}(\bar{\Omega})$ to (3.2) and for each $\lambda \in(0,1)$. Then the boundary value problem (1.1) has at least one solution $u \in C^{1, \alpha}(\bar{\Omega})$ with $\|u\|_{0} \leq r$.

We note that Theorem 3.1 not only guarantees the existence of a solution, but also gives information about its localization. Since the proofs of Theorem 3.1(1)-(3) are identical, we shall just prove Theorem 3.1(3). Firstly, we recall the following well-known results:

Lemma 3.1 (Nonlinear alternative of Leray-Schauder, [24]) Let $B[0, r]$ denote the closed ball in a Banach space $E$ with radius $r$, and let $T: B[0, r] \rightarrow E$ be a compact operator. Then either

(i) the equation $\lambda T u=u$ has a solution in $B[0, r]$ for $\lambda=1$, or

(ii) there exists an element $u \in E$ with $\|u\|_{E}=r$ satisfying $\lambda$ Tu $=u$ for some $0<\lambda<1$.

Proof of Theorem 3.1(3) According to Proposition 2.3, the operator $K$ from $L^{\infty}(\Omega)$ to $C^{1}(\bar{\Omega})$ is well defined and compact. We shall apply the nonlinear alternative of LeraySchauder to $E$ and to the operator $T: E \rightarrow E$, with $T u=K F u$, where $F: C(\bar{\Omega}) \rightarrow C(\bar{\Omega})$ is given by $(F u)(x)=f(x, u(x))$. On the other hand, it is clear that the fixed points of $T$ are the solutions of problem (1.1). Now the conclusion follows from Lemma 3.1 since condition (ii) is excluded by hypothesis.

Theorem 3.1 immediately yields the following existence and localization results.

Corollary 3.1 (1) Assume that $f$ satisfies (2.1), $p(x) \in C_{+}(\bar{\Omega})$ and there exist nonnegative continuous functions $a(x), b(x)$ and a continuous nondecreasing function $\psi: \mathbb{R}^{+} \rightarrow \mathbb{R}^{+}$such 
that

$$
|f(x, u)| \leq a(x) \psi(|u|)+b(x), \quad \forall(x, u) \in \Omega \times \mathbb{R} .
$$

Suppose, in addition, that there exists a real number $r>0$ such that

$$
r \geq(\psi(r)+1)^{\frac{1}{p^{-}-1}}|K(c(x))|_{L^{q(x)}(\Omega)},
$$

where $c(x)=\max \{a(x), b(x)\}=\frac{1}{2}(a(x)+b(x)+|a(x)-b(x)|)$. Then the boundary value problem (1.1) has at least one solution in $W_{0}^{1, p(x)}(\Omega)$ with $|u|_{L^{q(x)}(\Omega)} \leq r$.

(2) Assume that $p(x)$ is log-Hölder continuous and (3.4) holds. Suppose, in addition, that there exists a real number $r>0$ such that

$$
r \geq(\psi(r)+1)^{\frac{1}{p^{-}-1}}\|K(c(x))\|_{0} .
$$

Then the boundary value problem (1.1) has at least one solution in $C^{0, \alpha}(\bar{\Omega})$ with $\|u\|_{0} \leq r$.

(3) Assume that $p(x)$ is Hölder continuous and (3.4), (3.6) hold. Then the boundary value problem (1.1) has at least one solution in $C^{1, \alpha}(\bar{\Omega})$ with $\|u\|_{0} \leq r$.

Since the proofs of Corollary 3.1(1)-(3) are identical, we shall just prove Corollary 3.1(3).

Proof of Corollary 3.1(3) In order to apply Theorem 3.1(3), we have to show that condition (3.3) holds true for any solution $u \in C^{1, \alpha}(\bar{\Omega})$ to (3.2). Assume $u \in C^{1, \alpha}(\bar{\Omega})$ is any solution to (3.2) for some $\lambda \in(0,1)$ with $\|u\|_{0}=r$. Then

$$
u=\lambda T u=\lambda K F u \text {. }
$$

Furthermore, for all $x \in \bar{\Omega}$, by the monotonicity of $K$ and Lemma 2.1, we have

$$
\begin{aligned}
|u| & =\lambda|K F u(x)| \\
& \leq \lambda|K(a(x) \psi(|u|)+b(x))| \\
& \leq \lambda|K(a(x) \psi(r)+b(x))| \\
& \leq \lambda|K(c(x)(\psi(r)+1))| \\
& \leq \lambda(\psi(r)+1)^{\frac{1}{p(\xi)-1}}|K(c(x))| \\
& \leq \lambda(\psi(r)+1)^{\frac{1}{p(\xi)-1}}\|K(c(x))\|_{0} \\
& \leq \lambda(\psi(r)+1)^{\frac{1}{p^{-}-1}}\|K(c(x))\|_{0} .
\end{aligned}
$$

Taking the supremum in the above inequality, we obtain

$$
\|u\|_{0} \leq \lambda(\psi(r)+1)^{\frac{1}{p^{-}-1}}\|K(c(x))\|_{0} .
$$

Therefore, $r \leq \lambda r<r$ since $\lambda \in(0,1)$ and $\|u\|_{0}=r$. This is a contradiction.

We note that condition (3.1) can be satisfied under some suitable conditions. 
Theorem 3.2 Suppose that $p(x) \in C_{+}(\bar{\Omega}), f: \Omega \times \mathbb{R} \rightarrow \mathbb{R}$ is a continuous function and satisfies

$$
\lim _{|s| \rightarrow+\infty} \frac{f(x, s)}{s^{p^{-}-1}}=0,
$$

uniformly with respect to $x \in \Omega$. Then the boundary value problem (1.1) has a solution $u \in W_{0}^{1, p(x)}(\Omega)$ with $|u|_{L^{p^{-}}(\Omega)}<r$ for some $r \in \mathbb{R}^{+}$.

Proof From (3.7) it follows that, for all $\varepsilon>0$, there exists $C_{\varepsilon}>0$ such that

$$
|f(x, s)| \leq C_{\varepsilon}+\varepsilon|s|^{p^{-}-1} .
$$

Then, according to Theorem 1.16 of [7], $f$ induces a Nemytsky operator on $X=L^{p^{-}}(\Omega)$, still denoted by $f$. Setting $T(u)=K f(u)$, according to Proposition 2.3, $T \in C(X, X)$ is compact and (1.1) can be written in the form $u=T u, u \in X$. Let us show that there exists $r>0$ such that the homotopy $h(t, u)=u-t T(u)$ is admissible in $B_{r}=\left\{u \in X:|u|_{L^{p^{-}}(\Omega)}<r\right\}$. Otherwise, there exist $u_{n} \in X$, with $\left|u_{n}\right|_{L^{p^{-}}(\Omega)} \rightarrow+\infty$, and $t_{n} \in[0,1]$ such that $u_{n}=t_{n} T\left(u_{n}\right)$. This is equivalent to $-\operatorname{div}\left(\left|\nabla u_{n}\right|^{p(x)-2} \nabla u_{n}\right)=t_{n} f\left(x, u_{n}\right)$, with $u_{n} \in W_{0}^{1, p(x)}(\Omega)$. Taking $u_{n}$ as a test function, using (3.8) and the fact that $t_{n} \leq 1$, we get

$$
\int_{\Omega}\left|\nabla u_{n}\right|^{p(x)} d x \leq t_{n} \int_{\Omega}\left|f\left(x, u_{n}\right) u_{n}\right| d x \leq C_{\varepsilon} \int_{\Omega}\left|u_{n}\right| d x+\varepsilon \int_{\Omega}\left|u_{n}\right|^{p^{-}} d x .
$$

Case 1. $\|u\| \geq 1$.

In this case, using the Hölder and Poincaré inequality, we deduce

$$
c_{0}^{p^{-}}\left|u_{n}\right|_{L^{p^{-}}(\Omega)}^{p^{-}} \leq C_{\varepsilon}\left|u_{n}\right|_{L^{p^{-}}(\Omega)}+\varepsilon\left|u_{n}\right|_{L^{p^{-}}(\Omega)}^{p^{-}},
$$

where $c_{0}$ is the embedding constant of $W_{0}^{1, p(x)} \hookrightarrow L^{p^{-}}(\Omega)$. If we take $\varepsilon$ such that $\varepsilon<c_{0}^{p^{-}}$, this equation implies that $\left\|u_{n}\right\|_{L^{p^{-}}(\Omega)} \leq C_{1}$, for some $C_{1}>0$, a contradiction.

Case 2. $\|u\| \leq 1$.

In this case, using the Hölder and Poincaré inequalities, we deduce

$$
c_{0}^{p^{+}}\left|u_{n}\right|_{L^{p^{-}}(\Omega)}^{p^{+}} \leq C_{\varepsilon}\left|u_{n}\right|_{L^{p^{-}}(\Omega)}+\varepsilon\left|u_{n}\right|_{L^{p^{-}}(\Omega)}^{p^{-}} \leq C_{\varepsilon}\left|u_{n}\right|_{L^{p^{-}}(\Omega)}+\varepsilon\left|u_{n}\right|_{L^{p^{-}}(\Omega)}^{p^{+}} .
$$

If we take $\varepsilon$ such that $\varepsilon<c_{0}^{p^{+}}$, this equation implies that $\left\|u_{n}\right\|_{L^{p^{-}}(\Omega)} \leq C_{2}$, for some $C_{2}>0$, a contradiction again.

Thus the homotopy $h(t, u)=u-t T(u)$ is admissible on the ball $B_{r}$. Using the homotopy invariance, it follows that $\operatorname{deg}\left(I-T, B_{r}, 0\right)=\operatorname{deg}\left(I, B_{r}, 0\right)=1$, and hence there exists $u \in B_{r}$ such that $u=T(u)$, giving rise to a solution of problem (1.1).

Remark 3.1 We note that the fact that the homotopy $h(t, u)=u-t T(u)$ is admissible in $B_{r}$ implies $|u|_{L^{p^{-}}(\Omega)} \neq r$ for any solution of (3.2).

Using Theorem 3.1, Theorem 3.2 and Remark 3.1, we easily get:

Corollary 3.2 Assume $p(x) \in C_{+}(\bar{\Omega})$ and $f$ satisfies (3.7), then the boundary value problem (1.1) has at least one solution $u \in W_{0}^{1, p(x)}(\Omega)$ with $|u|_{L^{p^{-}}(\Omega)} \leq r$ for some $r>0$. 


\section{Existence and uniqueness via the Galerkin method}

In this section, we shall use the Brouwer fixed point theorem and the Galerkin method to prove the existence of a solution for problem (1.1).

Theorem 4.1 Assume that there exist constant $a>0$ and $\alpha(x) \in C_{+}(\bar{\Omega})$ with $\alpha^{+}<p^{-}$such that

$$
f(x, u) u \leq a\left(1+|u|^{\alpha(x)}\right), \quad \forall x \in \Omega, u \in \mathbb{R}
$$

with

$$
a<\frac{1}{\mathcal{C}^{\alpha^{+}}}
$$

where $\mathcal{C}$ is the embedding constant of $W_{0}^{1, p(x)}(\Omega) \hookrightarrow L^{\alpha(x)}(\Omega)$. Then problem (1.1) has at least one weak solution. Besides, any solution u of (1.1) satisfies the estimate

$$
\|u\| \leq R_{1}:=\max \left\{\left(\frac{a|\Omega|}{1-\mathcal{C}^{\alpha^{+}}}\right), 1\right\} .
$$

Proof Because $W_{0}^{1, p(x)}(\Omega)$ is a reflexive and separable Banach space, there exist $\left\{e_{j}\right\} \subset$ $W_{0}^{1, p(x)}(\Omega)$ and $\left\{e_{j}^{*}\right\} \subset\left(W_{0}^{1, p(x)}(\Omega)\right)^{*}$ such that

$$
X=\overline{\operatorname{span}\left\{e_{j}: j=1,2, \ldots\right\}}, \quad X^{*}=\overline{\operatorname{span}\left\{e_{j}^{*}: j=1,2, \ldots\right\}},
$$

and

$$
\left\langle e_{i}, e_{j}^{*}\right\rangle= \begin{cases}1, & i=j, \\ 0, & i \neq j .\end{cases}
$$

For convenience, let us put

$$
V_{n}=\operatorname{Span}\left\{e_{1}, \ldots, e_{n}\right\}
$$

Then $V_{n}$ is isometric to $\mathbb{R}^{n}$. In fact, each $v \in V_{n}$ is uniquely associated to $\eta=\left(\eta_{1}, \ldots, \eta_{n}\right) \in$ $\mathbb{R}^{n}$ by the relation $v=\sum_{k=1}^{n} \eta_{k} e_{k}$. We search for solutions $u_{n} \in V_{n}$ of the approximate problem

$$
\int_{\Omega}\left|\nabla u_{n}\right|^{p(x)-2} \nabla u_{n} \nabla e_{k} d x=\int_{\Omega} f\left(x, u_{n}\right) e_{k} d x, \quad k=1,2, \ldots, n .
$$

To solve this algebraic system, we define the operator $P_{n}: \mathbb{R}^{n} \rightarrow \mathbb{R}^{n}$,

$$
\left(P_{n} u\right)_{k}=\int_{\Omega}|\nabla u|^{p(x)-2} \nabla u \nabla e_{k} d x-\int_{\Omega} f(x, u) e_{k} d x, \quad u \in V_{n} .
$$

We note that $P_{n}$ is continuous from the continuity of $f(x, u)$ with respect to $u$. Therefore, we can use the following form of the Brouwer fixed point theorem: if there exists $R>0$ such that $\left\langle P_{n} u, u\right\rangle \geq 0$ whenever $\|u\|=R$, then $P_{n}$ has a root $u$ satisfying $\|u\| \leq R$ (see, 
e.g., [25]). From (4.1), Proposition 2.1 and the Poincaré inequality, we have for $u \in V_{n}$ with $\|u\| \geq 1$

$$
\begin{aligned}
\left\langle P_{n} u, u\right\rangle & \geq\|u\|^{p^{-}}-\int_{\Omega} f(x, u) u d x \\
& \geq\|u\|^{p^{-}}-a \mathcal{C}^{\alpha^{+}}\|u\|^{\alpha^{+}}-a|\Omega| .
\end{aligned}
$$

This shows, from (4.2), the existence of $R>1$, depending only on $\mathcal{C}, a$ and $|\Omega|$, such that $\left\langle P_{n} u, u\right\rangle \geq 0$ if $\|u\|=R$. Then system (4.4) has a solution $u_{n} \in V_{n}$ satisfying

$$
\left\|u_{n}\right\| \leq R, \quad \forall n \in \mathbb{N} .
$$

From this estimate, going to a subsequence if necessary, there is $u$ such that

$$
u_{n} \rightarrow u \quad \text { weakly in } W_{0}^{1, p(x)}(\Omega) \text {. }
$$

Besides, since $W_{0}^{1, p(x)}(\Omega) \rightarrow L^{\alpha(x)}(\Omega)$ compactly and the Nemytsky map $N_{f}$ is continuous from $L^{\alpha(x)}(\Omega)$ to $L^{\alpha^{\prime}(x)}(\Omega)$ (see [7]). Then fixing $k$ in (4.4) and letting $n \rightarrow+\infty$, we conclude that

$$
\int_{\Omega}|\nabla u|^{p(x)-2} \nabla u \nabla e_{k} d x=\int_{\Omega} f(x, u) e_{k} d x, \quad k=1,2,3, \ldots
$$

From the completeness of $\left\{e_{k}\right\}$, identity (4.5) holds with $e_{k}$ replaced by any $\varphi \in W_{0}^{1, p(x)}(\Omega)$, we get

$$
\int_{\Omega}|\nabla u|^{p(x)-2} \nabla u \nabla \varphi d x=\int_{\Omega} f(x, u) \varphi d x,
$$

which shows that $u$ is in fact a solution of problem (1.1). Finally, if $u$ is any solution of problem (1.1), then $\int_{\Omega}|\nabla u|^{p(x)} d x=\int_{\Omega} f(x, u) u d x$. Therefore, either $\|u\| \leq 1$ or

$$
\|u\|^{p^{-}} \leq a \mathcal{C}^{\alpha^{+}}\|u\|^{\alpha^{+}}+a|\Omega| \leq a \mathcal{C}^{p^{-}}\|u\|^{\alpha^{+}}+a|\Omega|
$$

and (4.3) follows.

Theorem 4.2 Let the assumptions of Theorem 4.1 hold, with (4.1) replaced by

$$
(f(x, u)-f(x, v))(u-v) \leq 0, \quad \forall x \in \Omega, \forall u, v \in \mathbb{R} .
$$

Then problem (1.1) has exactly one solution.

Proof Taking $v=0$ in (4.7), we get

$$
f(x, u) u \leq f(x, 0) u \leq \max _{x \in \bar{\Omega}}|f(x, 0)|+\max _{x \in \bar{\Omega}}|f(x, 0)||u|^{\alpha(x)}
$$

Setting $a:=\max _{x \in \bar{\Omega}}|f(x, 0)|$ in (4.8), we get (4.1). Hence, the existence part follows from Theorem 4.1. Now let $u$ and $v$ be two solutions of problem (1.1). Putting $w=u-v$, by (4.7) 
and Theorem 3.1 of [19], we have

$$
0 \leq \int_{\Omega}\left(|\nabla u|^{p(x)-2} \nabla u-|\nabla v|^{p(x)-2} \nabla v\right) \nabla w d x=\int_{\Omega}(f(x, u)-f(x, v)) w d x \leq 0 .
$$

We conclude that $w \equiv 0$, and hence $u=v$.

Competing interests

We declare that we have no competing interests.

\section{Authors' contributions}

GD conceived of the study, participated in its design and coordination, and helped to draft the manuscript. SY participated in the design of the study. All authors read and approved the final manuscript.

\section{Acknowledgements}

The authors are very grateful to the anonymous referees for their valuable suggestions. Research supported by the NSFC (No. 11261052, No. 11126296).

\section{Received: 8 June 2012 Accepted: 22 August 2012 Published: 7 September 2012}

\section{References}

1. Rüžička, M: Electro-rheological Fluids: Modeling and Mathematical Theory. Springer, Berlin (2000)

2. Zhikov, W: Averaging of functionals of the calculus of variations and elasticity theory. Math. USSR, Izv. 9, 33-66 (1987)

3. Antontsev, SN, Shmarev, SI: A model porous medium equation with variable exponent of nonlinearity: existence, uniqueness and localization properties of solutions. Nonlinear Anal. 60, 515-545 (2005)

4. Antontsev, SN, Rodrigues, JF: On stationary thermo-rheological viscous flows. Ann. Univ. Ferrara, Sez. 7: Sci. Mat. 52, 19-36 (2006)

5. Chen, Y, Levine, S, Rao, M: Variable exponent, linear growth functionals in image restoration. SIAM J. Appl. Math. 66(4), 1383-1406 (2006)

6. Diening, L, Hästö, P, Nekvinda, A: Open problems in variable exponent Lebesgue and Sobolev spaces. In: Drábek, P, Rákosník, J (eds.) FSDONA04 Proceedings, Milovy, Czech Republic, pp. 38 -58 (2004)

7. Fan, $X L$, Zhao, D: On the spaces $L^{p(x)}$ and $W^{m, p(x)}$. J. Math. Anal. Appl. 263, 424-446 (2001)

8. Harjulehto, P, Hästö, P: An overview of variable exponent Lebesque and Sobolev spaces. In: Herron, D (ed.) Future Trends in Geometric Function Theory, pp. 85-93. Univ. Jyväskylä, Jyväskylä (2003)

9. Samko, S: On a progress in the theory of Lebesgue spaces with variable exponent maximal and singular operators. Integral Transforms Spec. Funct. 16, 461-482 (2005)

10. Zhikov, WV (=V.V. Jikov), Kozlov, SM, Oleinik, OA: Homogenization of Differential Operators and Integral Functionals. Springer, Berlin (1994). (Translated from the Russian by GA Yosifian)

11. Zhikov, W: On some variational problems. Russ. J. Math. Phys. 5, 105-116 (1997)

12. Dai, G: Three symmetric solutions for a differential inclusion system involving the $(p(x), q(x))$-Laplacian in $\mathbb{R}^{N}$. Nonlinear Anal. 71, 1763-1771 (2009)

13. Dai, G: Infinitely many solutions for a Neumann-type differential inclusion problem involving the $p(x)$-Laplacian. Nonlinear Anal. 70, 2297-2305 (2009)

14. Dai, G: Infinitely many solutions for a hemivariational inequality involving the $p(x)$-Laplacian. Nonlinear Anal. 71, 186-195 (2009)

15. Dai, G: Three solutions for a Neumann-type differential inclusion problem involving the $p(x)$-Laplacian. Nonlinear Anal. 70, 3755-3760 (2009)

16. Dai, G: Infinitely many solutions for a differential inclusion problem in $\mathbb{R}^{N}$ involving the $p(x)$-Laplacian. Nonlinear Anal. 71, 1116-1123 (2009)

17. Fan, XL, Zhao, D: A class of De Giorgi type and Hölder continuity. Nonlinear Anal. 36, 295-318 (1996)

18. Fan, XL: On the sub-supersolution methods for $p(x)$-Laplacian equations. J. Math. Anal. Appl. 330, 665-682 (2007)

19. Fan, XL, Zhang, QH: Existence of solutions for $p(x)$-Laplacian Dirichlet problems. Nonlinear Anal. 52, 1843-1852 (2003)

20. Fan, $X L$, Han, $X Y$ : Existence and multiplicity of solutions for $p(x)$-Laplacian equations in $\mathbb{R}^{N}$. Nonlinear Anal. 59, $173-188(2004)$

21. Fan, XL: Global $C^{1, \alpha}$ regularity for variable exponent elliptic equations in divergence form. J. Differ. Equ. 235, 397-417 (2007)

22. Gilbarg, D, Trudinger, N: Elliptic Partial Differential Equations of Second Order. Springer, Berlin (1983)

23. O'Regan, D, Precup, R: Theorems of Leray-Schauder Type and Applications. Gordon and Breach, Amsterdam (2001)

24. Zeidler, E: Nonlinear Functional Analysis: Part I. Springer, New York (1985)

25. Kesavan, S: Topics in Functional Analysis and Applications. Wiley, New York (1989)

26. Moussaoui, T, Precup, R: Existence results for semilinear elliptic boundary value problems via topological methods. Appl. Math. Lett. 22, 126-129 (2009)

27. Ngô, QA: Existence results for quasilinear elliptic boundary value problems via topological method. arXiv:0805.0075v1 [math.AP] (2008) 